\title{
The Effectiveness of Cannabis and Cannabis Derivatives in Treating Lower Back Pain in the Aged Population: A Systematic Review
}

\author{
Helen Senderovich ${ }^{a}$ Hayley Wagman ${ }^{b}$ Dennis Zhang ${ }^{c}$ Danusha Vinoraj $^{d}$ \\ Sarah Waicus ${ }^{\mathrm{e}}$ \\ aDepartment of Family and Community Medicine, Division of Palliative Care, University of Toronto, Geriatrics, \\ Palliative Care, Pain Medicine, Baycrest, Toronto, ON, Canada; ${ }^{b}$ Wilfred Laurier University, Waterloo, ON, Canada; \\ 'Department of Family Medicine, University of British Columbia, Victoria, BC, Canada; 'Department of Medicine,

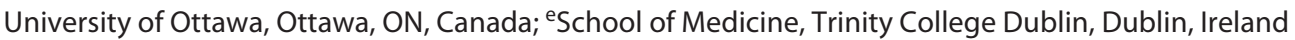

\section{Keywords \\ Lower back pain · Cannabis · Tetrahydrocannabinol · \\ Cannabidiol · Dronabinol · Aged population}

\begin{abstract}
Background/Aims: Cannabis is increasingly used in the management of pain, though minimal research exists to support its use since approval. Reduction in stigma has led to a growing interest in pharmaceutical cannabinoids as a possible treatment for lower back pain (LBP). The objective of this review was to assess the role and efficacy of cannabis and its derivatives in the management of LBP and compile global data related to the role of cannabis in the management of LBP in an aging population. Methods: A systematic review was conducted using predetermined keywords by 3 independent researchers. Predetermined inclusion and exclusion criteria were applied, and 23 articles were selected for further analysis. Results: Studies identified both significant and insignificant impacts of cannabis on LBP. Contradicting evidence was noted on the role of cannabis in the management of anxiety and insomnia, 2 common comorbidities with LBP. The existing literature suggests that cannabis may be used in the management of LBP and comorbid symptoms. Conclusions: Further research is needed to consider cannabis as an independent management option.
\end{abstract}

There is a lack of evidence pertaining to the benefits of cannabis in an aged population, and thus, additional research is warranted to support its use in the aged population.

(c) 2021 The Author(s)

Published by S. Karger AG, Basel

\section{Background}

Lower back pain (LBP) is a leading cause of disability globally, with damaging economic implications [1]. The 2010 Global Burden of Disease Study defined LBP as pain between the regions of the 12 th rib and lower gluteal folds [2]. There is a positive association between LBP and an aging population. The aged population is defined as individuals between 65 and 79 years of age. LBP was ranked the highest of 291 conditions ranked regarding disability in the global burden of disease, surpassing the previous ranking completed $<10$ years prior [2]. In 1990, the USA estimated the economic burden of back pain as over 24 billion dollars, 3.2\% of health-care costs [3]. Insomnia and anxiety are common comorbidities, reducing the quality of life and exacerbating the severity of $\operatorname{LBP}[4,5]$. Acute LBP is defined as 6-12 weeks of often nonspecific pain that spreads down one or both legs [6]. Persistent pain for a period of at least 3 months is termed chronic LBP [6]. In patients with dementia, this pain can be as-
(C) 2021 The Author(s)

Published by S. Karger AG, Basel

This is an Open Access article licensed under the Creative Commons Attribution-NonCommercial-4.0 International License (CC BY-NC) (http://www.karger.com/Services/OpenAccessLicense), applicable to the online version of the article only. Usage and distribution for commercial purposes requires written permission.
Correspondence to:

Helen Senderovich, hsenderovich @ baycrest.org 
sociated with increased agitation and behavioral symptoms [7]. LBP can be categorized as organic or mechanical pain. Organic pain is caused by disease, while mechanical pain results from damage to the spinal joints, discs, vertebrae, or soft tissues [8]. Over 90\% of LBP is mechanical [9]. Neuropathy arises from injury to nerve roots and is present in up to $55 \%$ of patients with chronic LBP [10, $11]$. Given the prevalence of LBP among the aged, exploration of the evidence surrounding alternative treatment options, such as cannabis, to minimize potential side effects imposed by conventional treatment plans is warranted. Moreover, it may support holistic management for the aged population.

\section{Pharmaceutical Formulations of Cannabis}

Marijuana has commonly been used to alleviate chronic pain. Routes of administration include oral, inhalation, sublingual, topical, or intravenous [12]. Medical marijuana is synthetically modified cannabis used for symptom management. Cannabis is a general term referring to the products derived from the plant genus Cannabis [13]. The active ingredients in plant-based cannabis, termed cannabinoids, are tetrahydrocannabinol (THC) the psychoactive component and cannabidiol (CBD). Cannabinoids are widely used in pain relief and may be synthetically modified for use [14]. Dronabinol is a synthetic form of THC with the same psychoactive and pain modulation properties as the plant counterpart [15]. Pharmaceutical cannabinoids include dronabinol (brand names Marinol $\odot$ and Syndros $\odot$ ); nabilone (a synthetic THC derivative with the brand name Cesamet( $)$ ); and nabiximols (oral spray containing THC and CBD also known as brand names Cesamet( $\odot$ and Epidiolex@) [16]. Globally, most cannabis-based pharmaceuticals are not approved for medical use. Nabilone, Epidiolex, and dronabinol are used in Canada and the USA, while nabiximols are used in Canada, New Zealand, and Europe. Marinol@ was recently withdrawn from the Canadian market by the manufacturer for unknown causes [17]. Cannabis also exists as oil and may be used in baked goods resulting in slower THC absorption, a delayed onset and a prolonged period of intoxication compared to inhalation [18]. The utilization of CBD in conditions such as epilepsy, multiple sclerosis (MS), Huntington's disease, and more, is currently being evaluated.

Marinol@ has been approved in the USA since 1985 for appetite stimulation in patients with HIV/AIDS and chemotherapy-associated nausea. Oral dronabinol exhibited little benefit in the treatment of chronic pain and failed to show much promise in the management of neurogenic pain in patients with MS [19]. With a bioavailability of 10$35 \%$, inhaled THC has a peak plasma concentration between 3 and $10 \mathrm{~min}$. However, due to hepatic first-pass metabolism, the bioavailability of THC was $6 \%$ with oromucosal administration. Peak blood concentrations were found to occur $1-5 \mathrm{~h}$ post-dose, with a slow and unpredictable onset [20]. Nabilone (Cesamet( )), a synthetic THC analog has been shown to exhibit analgesic effects in neuropathic pain. The beneficial properties of nabilone may be attributed to its prolonged half-life and increased potency. Another synthetic THC analog, ajulemic acid (CT3, IP751), has also shown improvement in neuropathic pain [20]. Cannador (IKF-Berlin), an orally administered pill composed of cannabis extract, has been shown to improve spasm-associated pain among patients with MS [20]. In terms of LBP treatment, management of spasm-related pain is commonly encountered in occupational injuries among laborers. Cannador may serve as an alternative treatment option for patients facing daily NSAID and salicylate consumption. Finally, Sativex, an oromucosal spray, is a combined THC:CBD formulation that acts as a CB1 partial agonist. Sativex was approved by Health Canada in June 2005 and August 2007 for neuropathic pain in MS and cancer pain unresponsive to opioids [20].

\section{Mechanisms of Action}

The human body contains 2 G-protein coupled receptors, CB1 and CB2. Binding of these G-protein-coupled receptors blocks the release of pain-inducing neurotransmitters in the central nervous system (CNS) [21]. The $\mathrm{CB} 1$ receptor is located in central and peripheral neurons, affecting cognition, memory, control of motor functions, and analgesia. The CB2 receptor is mainly found in immune cells, altering cytokine release and cell migration in the central and peripheral nervous systems. THC is the main bioactive ingredient in cannabis. THC binds CB1 and $\mathrm{CB} 2$ receptors with equal affinity mimicking the effects of endogenous cannabinoids [22, 23]. Binding these 2 receptors may attenuate neuropathic pain [24]. CB2 activation is shown to suppress acute, chronic, and neuropathic pain by stimulating the release of $\beta$-endorphin from peripheral keratinocytes $[25,26]$. Endogenous endorphins subsequently act on afferent nociceptors to inhibit pain transmission. In LBP, signals from nociceptors are sent to the spinal dorsal horn and ultimately sent through ascending pathways toward somatosensory cor- 
Table 1. Side effects of cannabis

\begin{tabular}{llll}
\hline Psychiatric & Neurological & Gastrointestinal & Cardiovascular \\
\hline Anxiety & Memory deficits & Xerostomia & Hypertension \\
Panic attacks & Learning deficits & Weight changes & Tachycardia \\
Agitation & Vertigo & Appetite changes & Vascular constriction \\
Palpitations & Drowsiness & Diarrhea & \\
PTSD & Catecholamine release & Cannabis hyperemesis syndrome & \\
& Ataxia & & \\
& Cognitive impairment & & \\
\hline
\end{tabular}

PTSD, post-traumatic stress disorder.

tices in the brain $[25,26]$. CBD does not act directly on either $\mathrm{CB} 1$ or $\mathrm{CB} 2$ receptors, instead encouraging the body to make use of its own cannabinoids [24]. CBD enhances the activity of 5HT1 a receptors to mediate anxiolytic and depressive symptoms. Additionally, CBD binds to inhibitory glycine a1 and a3 receptors attenuating excitatory neuronal pathways in the CNS [23]. Finally, recent studies suggest the involvement of CBD and inhibition of the adenosine receptor $\mathrm{A} 2 \mathrm{~A}$, reducing adenosine transport [20]. The inhibition of adenosine, a well-known participant in pain and inflammatory pathways, may be another mechanism of analgesia.

THC and CBD demonstrate potential for the treatment of LBP. The neurotransmitter and cytokine inhibitory actions of THC, with the serotonergic and neuroexcitatory inhibiting properties of $\mathrm{CBD}$, provide a new, plausible, means for pain management. Though THC and CBD exert separate analgesic effects, they are commonly administered together. THC inhibits PGE-2 synthesis, platelet aggregation, and stimulation of lipoxygenase, attenuating pain [19]. A major difference between THC and CBD is the psychotic-like symptoms that can be produced at high THC levels [27]. CBD is often used in conjunction with THC as hepatic first-pass metabolism of THC to 11-hydroxy-THC, the more psychoactive form, is inhibited [28]. As a result, CBD may act as a moderator of negative psychotic effects induced by THC.

\section{Side Effects of Cannabis}

A systematic review found cannabis to worsen psychiatric disorders and be positively correlated with anxiety, panic attacks, and cognitive impairments (memory and learning deficits) among patients being treated for noncancerous pain [29, 30]. Vertigo, drowsiness, ataxia, and an increase in violent outbursts were report- ed in patients with post-traumatic stress disorder [31, 32]. Sensations of euphoria and perceptual distortion were side effects in patients with chronic pain and anxiety [33]. Changes in weight and appetite were also noted [34]. Less commonly, long-term cannabis use may lead to cannabis hyperemesis syndrome: cyclical vomiting, abdominal pain, and dehydration [35]. Dried cannabis can cause hypertension, tachycardia, catecholamine release, and vascular constriction [33]. There have been occasional reports of adverse cardiovascular events; however, healthy individuals have minimal risk compared to older individuals with a history of similar complications [21, 36, 37]. Smoking cannabis, a possible risk factor for lung cancer, can lead to chronic bronchitis $[33,38]$. However, CBD suppresses cancer growth, kills cancer cells, and prevents cancerous cells from invading tissues and spreading in the body; however, there is a paucity of safe and effective clinical trials assessing potential risk and benefits $[33,39,38]$. A patient-reported study focused on aged patients (patients older than 65 years) found that dizziness was one of the most common adverse effects of cannabis use [40]. Cognitive impairment and agitation has also been associated in aged individuals, especially with neurodegenerative comorbidities such as dementia [7]. This is particularly concerning in a frail population where dizziness may potentiate falls.

Many chronic pain investigations have shown nonsignificant reductions in pain intensity, particularly in patients with nonneuropathic pain [12]. Cardiovascular events were recorded after cannabis use in young people, and high doses noted to precipitate myocardial infarction [41]. However, there is little evidence to support the relationship between cannabis and myocardial infarctions in the absence of pre-existing cardiac disease. Cannabis use has been correlated with anxiety, panic attacks, worsening of existing psychiatric disorders, and insom- 
Fig. 1. PRISMA flow diagram: search history. RCT, randomized controlled trial.

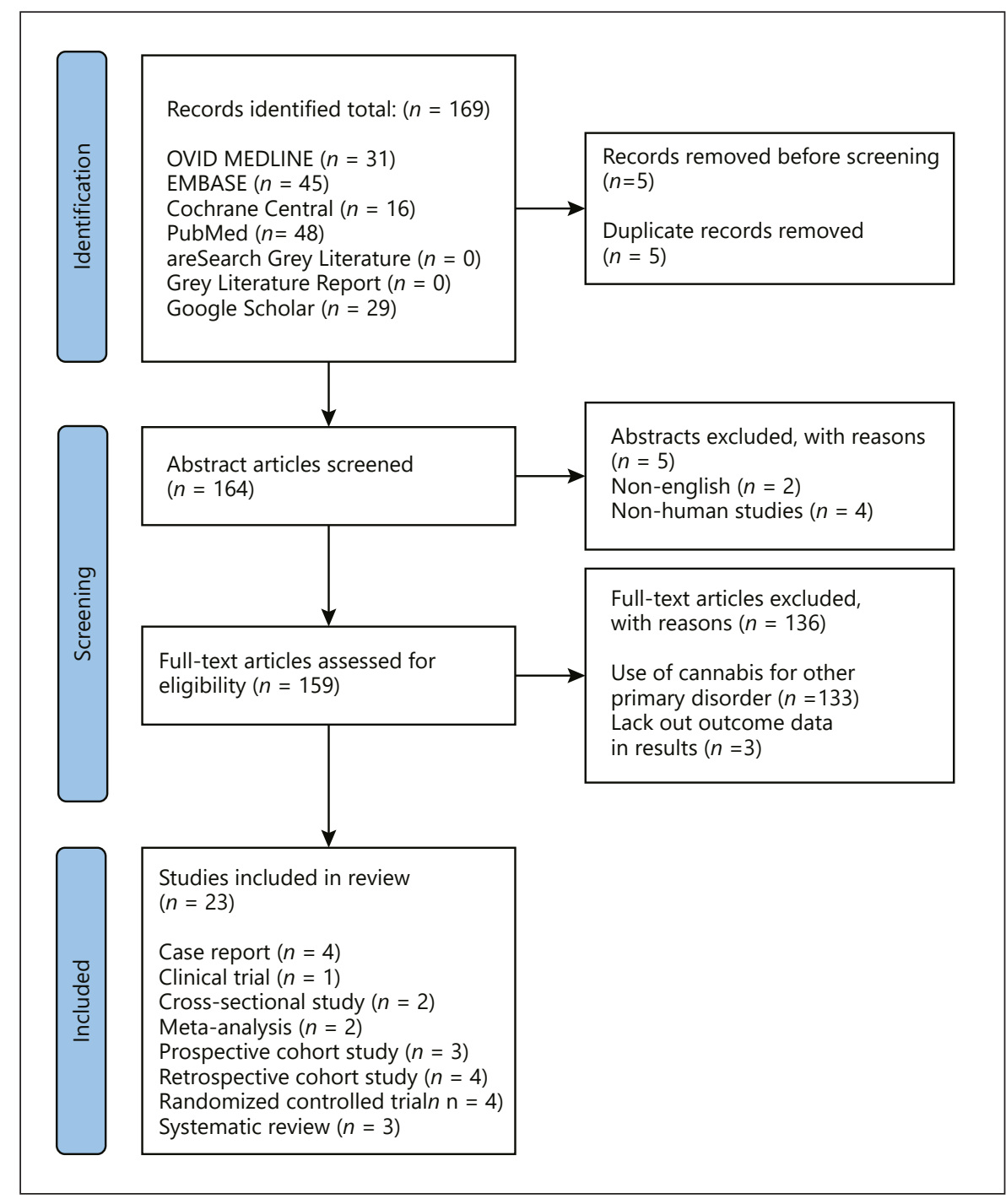

nia, all of which commonly coexist in patients with LBP [29]. Acute effects of cannabis include cognitive impairment and neurocognitive deficits in learning and memory [31]. Euphoria and perceptual distortions were also noted [42]. While evidence exists supporting the use of pure $\mathrm{CBD}$, and not THC, in the treatment of anxiety associated with chronic pain, there is ongoing controversy [32]. The risk of cognitive impairment is greater when THC enters the CNS rapidly in high concentrations as observed when this derivative is smoked or vaporized. Moreover, there is currently no evidence to support the use of dried cannabis for pain treatment and should only be considered in patients suffering from neuropathic pain and nonresponsive to standard treatment modalities [43]. Side effects of cannabis and its derivatives are summarized in Table 1.

Cannabis in Treating Lower Back Pain

\section{Objectives}

This systematic review aimed to provide a comprehensive understanding of the current landscape of cannabis, its role in LBP management, and explore the following:

1. The role of cannabis in LBP management in aged populations

2. The difference in structure, mechanism of action, and efficacy of THC and CBD

3. The impact of existent routes of administration on the effectiveness of cannabis

4. The outcomes of cannabis utilization and its side-effects while managing LBP 


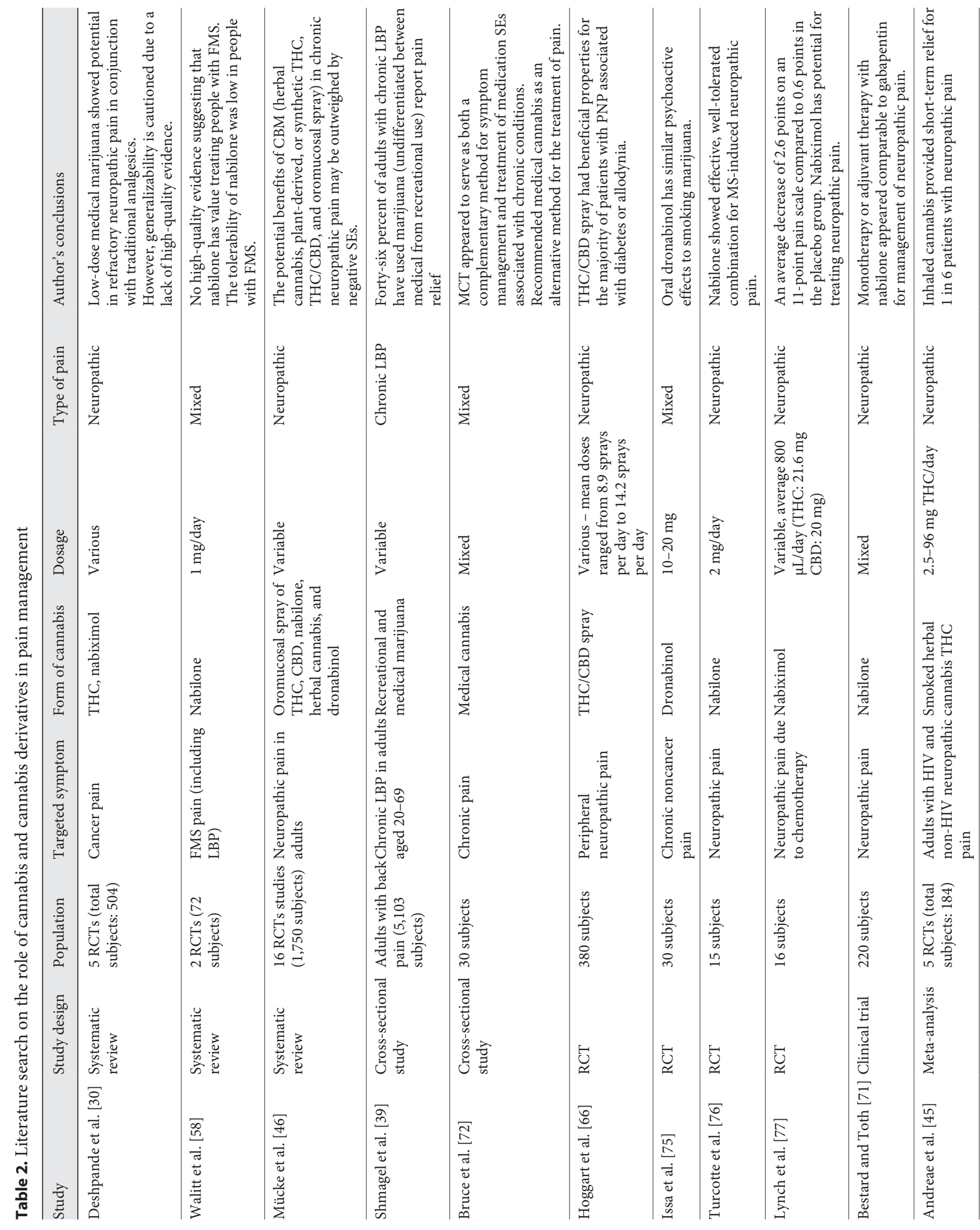




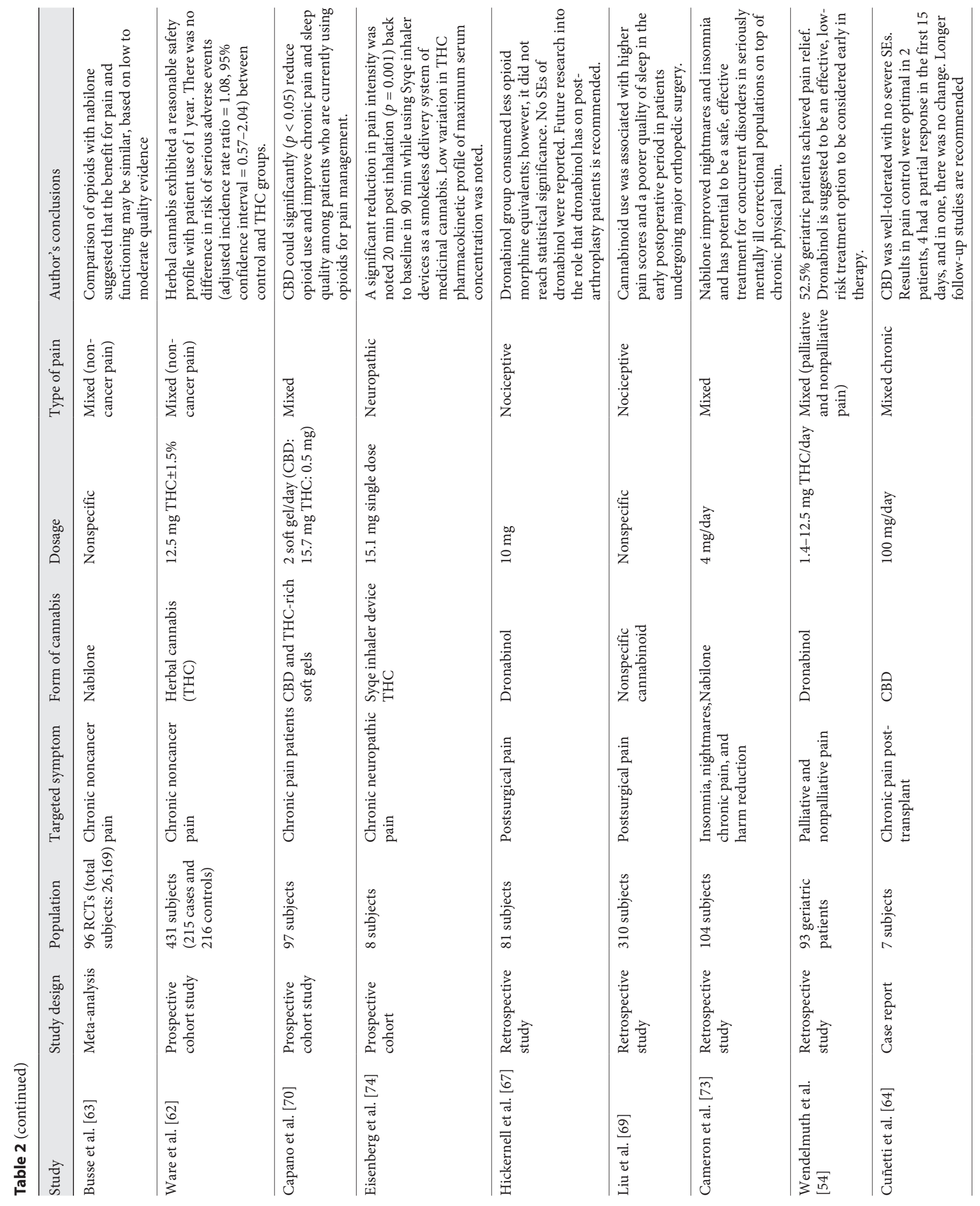



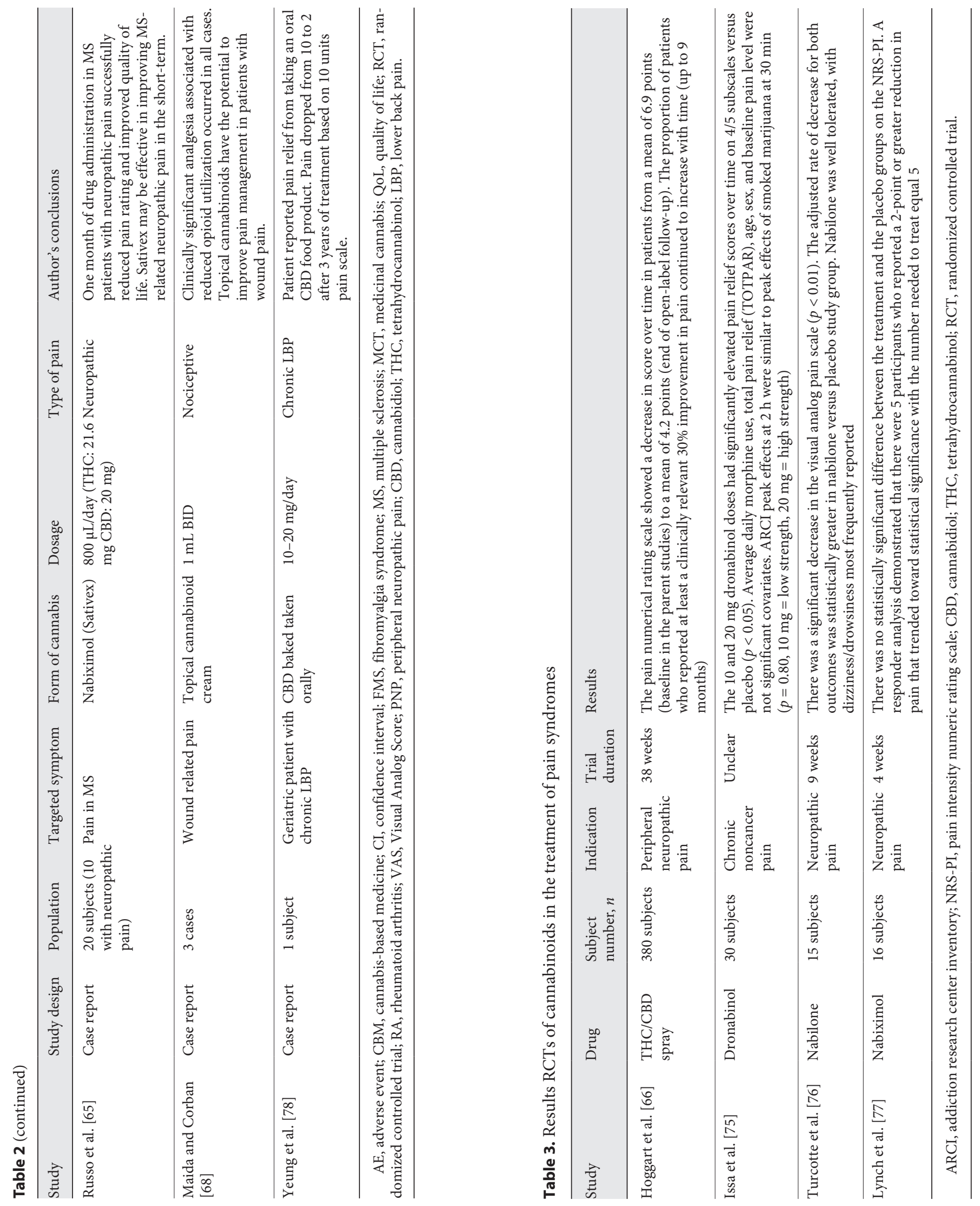


\section{Methods}

A systematic review was conducted by 3 independent researchers utilizing PubMed, OVID MEDLINE, Cochrane Central, and EMBASE. Additional non-peer-reviewed sources were searched, including Google Scholar, CareSearch Grey Literature, and Grey Literature Report. Eligibility assessment was performed by independent reviewers, and disagreements were resolved by consensus. A data extraction excel sheet was developed to compile and summarize relevant studies. Duplicates were removed before applying the inclusion and exclusion criteria. Inclusion criteria were established in line with the study objective, where relevant articles underwent data extraction and analysis.

\section{Inclusion Criteria}

Articles written in English language from January 2010 to September 2020, with an emphasis on the use of cannabis or its derivatives for the management of LBP, statistically significant human studies with $(p<0.05)$, and studies that explored chronic pain in older adults were included. The PICO tool was used to guide the keywords used in the search. All study designs were evaluated. The MeSH term aged is defined as "a person 65 years or older." A full search history can be found in Figure 1. The keywords used are as follows: "Low Back Pain," "THC," "CBD," "Cannabis," "Dronabinol," and "Cannabidiol" and "Aged."

\section{Exclusion Criteria}

Studies with nonhuman subjects, written in a language other than English, used cannabinoids for a primary purpose other than pain management, those reporting no outcomes, and duplicates between databases were excluded.

\section{Search Results}

Relevant articles underwent data extraction and analysis. A search yielded 169 references (31 in Medline, 45 in Embase, 16 in Cochrane Central, 48 in PubMed, 29 in Google Scholar, 0 in CareSearch Grey Literature, and 0 in Grey Literature Report) that matched predefined search parameters. Five articles were removed before abstract screening due to duplication. 164 article abstracts were screened, 2 were excluded as non-English, and 4 were excluded as nonhuman studies. There were 159 full-text articles assessed with 133 articles being excluded as cannabis treatment was being used for another primary disorder other than LBP, and 3 articles were excluded for a lack of reported outcome. There were 23 articles that met the final inclusion criteria, which included 3 case reports, 1 clinical trial, 2 cross-sectional study, 2 meta-analyses, 3 prospective cohort studies, 4 retrospective cohort studies, 4 randomized controlled trials (RCTs), and 3 systematic reviews found in Figure 1. Demographic (e.g., age range, sample size, and symptoms), cannabis treatment (form of cannabis, dosage, and duration), and the outcomes (pain relief and side effects) were extracted from reviewed studies. Pain reduction was the primary measure of efficacy in the studies. Corroborative themes were also identified and the authors responsible for the contributing research were cited and summarized in Tables 2 and 3. The Cochrane Risk of Bias Tool was used in reviewed studies to assess bias in RCTs found in Table 4.

\section{Results}

\section{Supporting Evidence for Cannabis Use in LBP}

Medical cannabis has been suggested to treat LBP acutely. A systematic review conducted by Deshpande et al. [30] found the majority of patients with neuropathic pain treated with medicinal cannabis had a clinically significant decrease in pain ( $>2$ points on a 10-point scale). Another systematic review examined a different form of cannabis such as oromucosal sprays containing THC, CBD, nabilone, herbal cannabis, and dronabinol used to treat adults, including the aged population with neuropathic pain suggested that all cannabis-based medicines pooled together reduced pain intensity and improved sleep problems and psychological distress compared with placebo [44].

Three of the 4 RCTs reported beneficial properties of cannabis and cannabis derivatives on LBP. Hoggart et al. [45] examined 380 patients with peripheral neuropathic pain administering oromucosal THC/CBD mixed spray over 38 weeks. There were clinically relevant improvements of at least 30\% throughout the 38-week study [45]. Mean doses ranged from 8.9 sprays to 14.2 sprays per day [45]. An RCT conducted by Issa et al. [46] examined 30 subjects with chronic noncancer pain administering 10 and $20 \mathrm{mg}$ of dronabinol revealed a significant decrease in pain $(p<0.05)$ compared to placebo. Turcotte et al. [47] treated 15 patients with $2 \mathrm{mg}$ /day of nabilone for neuropathic pain revealed that nabilone had a significant group and time interaction in decreasing pain, measured by the Visual Analog Scale (VAS) for pain $(p<0.01)$ when compared to placebo (Table 3 ).

A meta-analysis conducted by Busse et al. [48] examined the efficacy of opioids in comparison to different treat- 
Table 4. Risk of bias in RCTs

\begin{tabular}{|c|c|c|c|c|c|c|}
\hline Study & \multicolumn{2}{|l|}{ Selection bias } & $\begin{array}{l}\text { Performance bias } \\
\text { blinding of participants } \\
\text { and personnel }\end{array}$ & $\begin{array}{l}\text { Detection bias } \\
\text { blinding of } \\
\text { outcome assessor }\end{array}$ & $\begin{array}{l}\text { Attrition bias } \\
\text { incomplete } \\
\text { outcome data }\end{array}$ & $\begin{array}{l}\text { Reporting bias } \\
\text { selective } \\
\text { reporting }\end{array}$ \\
\hline Hoggart et al. [66] & Low & High & High & High & High & Low \\
\hline Issa et al. [75] & Low & Low & High & Low & Low & Low \\
\hline Turcotte et al. [76] & Low & Low & Low & Low & Low & Low \\
\hline
\end{tabular}

RCT, randomized controlled trial.

ments such as nonsteroidal anti-inflammatory drugs and nabilone. They found evidence suggesting only minimal difference between opioids and nabilone efficacy for pain relief $(p=0.77)$ when measured using the VAS for pain [48].

Three prospective cohort studies examined different forms of cannabis found different levels of efficacy in relieving pain. Capano et al. [49] examined CBD- and THCrich soft gels in 97 patients with chronic pain and found CBD could significantly $(p<0.05)$ reduce opioid use and improve chronic pain and sleep quality. Ware et al. [50] who examined the safety profile of herbal cannabis compared to controls who did not use cannabis for $431 \mathrm{pa}-$ tients with chronic pain and concluded that herbal cannabis had a reasonable safety profile and higher pain relief over a 1-year range. Eisenberg et al. [51] who examined THC use with an inhaler device in 8 patients with chronic neuropathic pain, revealed a significant reduction in pain intensity 20 min post-inhalation $(p=0.001)$ which was back to baseline in $90 \mathrm{~min}$.

When examining geriatric populations exclusively, a retrospective study conducted by Wendelmuth et al. [52] specifically assessed palliative care populations with chronic pain treated with dronabinol and revealed that $52.5 \%$ of geriatric patients achieved pain relief over a range of 1.5-12.5 mg of THC per day. Additional comorbid mental health disorders were assessed by Cameron et al. [53] when nabilone was administered for chronic pain. It was found that $4 \mathrm{mg} /$ day of nabilone in addition to relief of pain also improved nightmares, insomnia, and other mental health disorders [53].

Multiple forms and doses of cannabis were assessed in 4 different case reports revealed different benefits. Russo et al. [54] who examined chronic neuropathic pain in 20 patients (10 had neuropathic pain) with MS administering nabiximol, revealed that 1 month of $800 \mu \mathrm{L} /$ day of nabiximol successfully reduced pain rating and improved the quality of life in all 10 subjects. Cuñetti et al. [55] who examined chronic post-transplant pain in 7 subjects who received 100 $\mathrm{mg}$ /day of CBD revealed that 6 out of 7 patients had a positive partial response in improvement of pain control [55]. Topical cream was assessed in 3 patients with nociceptive pain by Maida and Corban [56] who revealed clinically significant analgesia $(p<0.05)$ associated with $1 \mathrm{~mL}$ of topical cannabinoid cream, which reduced opioid utilization in all 3 patients. An alternative oral form where $10-20 \mathrm{mg}$ of CBD baked into a food product was assessed in one 87-year-old geriatric patient with chronic LBP by Yeung et al. [57] who observed pain relief from $10 / 10$ on a pain scale to $2 / 10$ after 3 years of treatment consuming baked goods of CBD. These studies provide promising evidence that chronic pain among the aged may be treated with a combination of lowdose opioid and cannabis, if not entirely with cannabis.

In general, medical cannabis is reported to be the highest utilized substance for LBP for adults, including the aged population [39]. A cross-sectional study examined chronic LBP in 5,103 individuals and found that $46 \%$ of individuals had used recreational cannabis for pain management [39]. A more recent cross-sectional study conducted by Bruce et al. [58] examined the use of medical cannabis on chronic pain relief in adults including the aged population and revealed that medical cannabis was effective and complementary to pharmacological analgesics in pain management and treatment of side effects associated with chronic pain.

\section{Opposing Evidence for Cannabis Use in LBP}

When analyzing the effects of THC solely, the negative side effects were more prominent than those in CBD treatment. A systematic review examined nabilone treatment for fibromyalgia, including LBP, finding conflicting results [59]. This critical review suggested that studies superficially claimed that nabilone is a safe treatment. There was a high prevalence of adverse side effects such as dizziness, nausea, dry mouth, and drowsiness not solely related to cannabis but also to other existent comorbidities 
in those affected with fibromyalgia. As a result, it was concluded that the efficacy of nabilone had no value in complex pain disorders [59]. The route of administration seemed to limit efficacy when cannabis was inhaled. A meta-analysis examined smoked herbal cannabis as a treatment for chronic neuropathic pain found only shortterm relief for 1 in 6 patients in HIV and non-HIV patients [60].

Chronic pain induced surgically revealed conflicting results with regards to response to cannabis. A retrospective study conducted by Hickernell et al. [61] examined the efficacy of dronabinol in postsurgical nociceptive pain for 80 patients who received $10 \mathrm{mg}$ of dronabinol revealed that there were fewer opioids needed for controlling the pain. However, this study did not reach statistical significance for the efficacy of dronabinol for pain relief compared to opioids. In contrary, Liu et al. [62] examined the response to cannabinoids of postsurgical pain revealed that cannabinoid use was associated with higher pain scores and a poorer quality of sleep in the early postoperative period in patients who underwent major lumbar orthopedic surgery.

\section{Risk of Bias}

The Cochrane Risk of Bias tool and the Jadad scales were used to assess risk of bias in included RCTs. This may have contributed to inaccuracies in the assessment of study quality. Pooled data from the 5 RCTs could not be statistically analyzed due to small sample sizes. Discrepancies between the study design and small sample sizes make it difficult to generate conclusions with certainty. The risk of bias can be seen in Table 4. With multiple forms and routes of administration for treatment of chronic pain with cannabinoids, there is concern around insufficient evidence as a result of inconsistency among studies reviewed and lack of a standardized approach to evaluate outcomes [63]. In general, preference for cannabis held by patients may exceed its clinical effectiveness.

\section{Discussion}

While our investigation provides some evidence on the effectiveness of cannabis and its derivative in the treatment of LBP and comorbid conditions, further research is required. Only 5 small RCTs $(n=214,12,17,4$, 2) reported data on the efficacy and safety of cannabinoids among patients older than 65 years, separately [64]. There appears to be greater benefit in the utilization of THC derivatives for the suppression of acute, chronic, and neuropathic pain in the management of malignant and nonmalignant pain, while CBD derivatives may be better suited for comorbid symptoms of anxiety and insomnia [45]. The negative psychoactive effects of THC has been documented in older adults, where agitation, dizziness, and fatigue are heightened, limiting the utilization of the THC for this group [57]. A lack of investigation into these benefits as they pertain to a geriatric population makes it difficult to conclude whether cannabis or its derivatives may benefit an aged population.

Compared to conventional treatments such as pharmacotherapy or physical therapy, a small analgesic benefit with the use of selective cannabinoids was seen in a review of current RCTs [65]. Cannabis extract is noted as a possible treatment in MS for the management of pain and muscle stiffness [66]. The findings are consistent with the current literature. An RCT of 30 patients aged 65 years who were treated with cannabis for LBP $>6$ months revealed a significant reduction in pain intensity and an improvement in the quality of life [40]. Cannabis may be trialed as adjunctive therapy for neuropathic pain, where there has been a failure of response to standard analgesic modalities (i.e., opioids, anticonvulsants, and antidepressants) [47]. Furthermore, a recent meta-analysis conducted by Velayudhan et al. [67] examined 6,217 patients aged 50 years and older on the safety and tolerability of cannabis and its derivatives. It was found that THC was associated with side effects and that THC and CBD combinations were less tolerable for patients older than 65 and 75 years; however, when results were pooled, it was generally concluded that cannabis-based medicines were safe to use in aged individuals [67].

\section{Limitations}

This review revealed a lack of available evidence on the role of cannabis and it's derivatives in older population. The outcomes described by some of the studies were not statistically significant. The reviewed trials were of short duration, and there was no standardized approach; variable dosages of cannabis and its derivatives were used, and it was noted a lack of appropriate reporting of outcome measures. Only a handful of identified studies exceeded 50 participants. These small sample sizes and short duration of treatment limited the statistical power of the reviewed studies and created difficulties with drawing the conclusions that could be generalized to the geriatric population. The lack of statistical significance and small sample size of some of the studies limit the ability to assess the true efficacy of cannabis in baked goods. Moreover, individuals older than 65 years were typically excluded from RCTs because the harm of 
novel drugs normally outweighs the benefit. Thus, no studies exclusively explored the use of cannabis and its derivatives in the geriatric population. Additionally, some studies did not focus solely on LBP. Disorders such as fibromyalgia and MS were driving LBP in some studies. This limited the ability to compare the cause of LBP.

\section{Future Directions}

Future studies should focus on comparing cannabinoids to the existing gold standard pain reduction techniques in lieu of previously comparing to the placebo. Differences in analgesic effects among various administration methods of THC and CBD along with recommended ratios are currently unclear and remain a research priority. Studies focusing on the role of cannabis in pain and anxiety reduction are warranted as current evidence is contradictory. Further studies are required to determine the benefits of the combined use of opioids and/or other drugs with cannabinoids in the treatment and management of neuropathic pain [68]. Research evaluating the effects of cannabinoids solely in geriatric populations, given a complete absence of high-quality studies is needed.

\section{Conclusion}

This review examined the efficacy of numerous types of cannabis for the management of LBP in the aged population. In general, pain is a multifaceted and subjective phenomenon. It is often difficult to quantify with questionnaires. THC derivatives such as dronabinol and nabilone revealed more side effects due its psychoactive chemical properties. Treatments with higher levels of CBD such as $\mathrm{CBD}$ and nabiximol revealed fewer negative psychotic effects. Negative side effects of CBD and THC included dizziness, nausea, dry mouth, and poor sleep quality. Special consideration is needed in older adults due to polypharmacy, slower pharmacokinetic properties, and comorbid conditions. However, the benefits may outweigh the risks when cannabinoids and its derivatives are administered especially in PC palliative care, where the goals of care are comfort, symptoms control, and improvement in the quality of the remaining life. Cannabinoids may be an effective, low-risk treatment for patients with chronic pain. Anxiolytic effects of cannabinoids in the aged population are important as it can reduce the need for opioids and other pharmacological analgesics. Alternative routes in addition to traditional inhalation showed success which included oral derivatives of cannabinoids, such as baked goods, topical creams, soft gels, and oromucosal sprays. All forms of cannabinoids have shown considerable pain reduction for aged patients. It is important to have various routes of administration to achieve clinically significant pain reduction which can be useful in aged population and in palliative care settings. Cannabinoids and its derivatives may be utilized as an alternative or adjuvant for chronic pain in aged people, especially when standard analgesic modalities alone have failed.

\section{Acknowledgements}

We would like to thank Vithusha Yogeswaran (BMSc), Mohammed Faizan Ul Haq (MD), Joshua Tordjman (MSc), Akash Yendamuri (MD), Kevin Gunawan (BSc), Pablo Merino Garduño (MD), Keisa Mokenela (BSc), and Katherine Majerovich (MSc) for their contributions in the preparation of this manuscript.

\section{Statement of Ethics}

The authors declare this research complies with the guidelines of the World Medical Association Declaration of Helsinki.

\section{Conflict of Interest Statement}

The authors have no conflicts of interest to declare.

\section{Funding Sources}

This research received no specific grant from any funding agency in the public, commercial, or not-for-profit sectors.

\section{Author Contributions}

Helen Senderovich was responsible for the conception, design, drafting, clinical revisions, and final approval of the version to be published. Helen Senderovich is accountable for all aspects of the published work. Hayley Wagman was responsible for drafting of the manuscript and interpretation of the data. Dennis Zhang was responsible for drafting of the manuscript and interpretation of the data. Danusha Vinoraj was responsible for drafting of the manuscript and interpretation of the data. Sarah Waicus was responsible for drafting of the manuscript, interpretation of the data, and critical revisions of the manuscript.

\section{Data Availability Statement}

All data generated or analyzed during this study are included in this article. Further enquiries can be directed to the corresponding author. 


\section{References}

1 Loney PL, Stratford PW. The prevalence of low back pain in adults: a methodological review of the literature. Phys Ther. 1999;79(4): 384-96.

2 Hoy D, March L, Brooks P, Blyth F, Woolf A, Bain $\mathrm{C}$, et al. The global burden of low back pain: estimates from the global burden of disease 2010 study. Ann Rheum Dis. 2014;73(6): 968-74.

3 Kent PM, Keating JL. The epidemiology of low back pain in primary care. Chiropr Osteopat. 2005;13(1):13.

4 Bramoweth AD, Renqvist JG, Germain A, Buysse DJ, Gentili A, Kochersberger G, et al. Deconstructing chronic low back pain in the older adult: step by step evidence and expertbased recommendations for evaluation and treatment: part VII: insomnia. Pain Med. 2016;17(5):851-63.

5 Carley JA, Karp JF, Gentili A, Marcum ZA, Reid MC, Rodriguez E, et al. Deconstructing chronic low back pain in the older adult: step by step evidence and expert-based recommendations for evaluation and treatment: part IV: depression. Pain Med. 2015;16(11): 2098-108.

6 Koes BW, Tulder MV. Acute low back pain. Am Fam Physician. 2006;74(5):803-5.

7 Mueller A, Fixen DR. Use of cannabis for agitation in patients with dementia. Sr Care Pharm. 2020;35(7):312-7.

8 Tiltmann K. Mechanical low back pain. Spine Universe; 2019 Aug 27. Available from: https: //www.spineuniverse.com/conditions/backpain/low-back-pain/your-low-back-painmechanical Accessed 2020 Sep.

9 Government of Ontario's Provincial Low Back Pain Strategy. Core back toll: a guide. 2012 Apr 1. Available from: http://www. health.gov.on.ca/en/pro/programs/ecfa/ docs/lb_tk_core_tool_guide_c.pdf Accessed 2020 Sep.

10 Baron R, Binder A, Attal N, Casale R, Dickenson $\mathrm{AH}$, Treede RD. Neuropathic low back pain in clinical practice. Eur J Pain. 2016; 20(6):861-73.

11 Kaki AM, El-Yaski AZ, Youseif E. Identifying neuropathic pain among patients with chronic low-back pain: use of the Leeds assessment of neuropathic symptoms and signs pain scale. Reg Anesth Pain Med. 2005;30(5):422-8.

12 Whiting PF, Wolff RF, Deshpande S, Di Nisio M, Duffy S, Hernandez AV, et al. Cannabinoids for medical use: a systematic review and meta-analysis. JAMA. 2015;313(24):2456-73.

13 Small E, Cronquist A. A practical and natural taxonomy for cannabis. Taxon. 1976;25(4): 405-35.

14 Atakan Z. Marijuana as medicine? The science beyond the controversy. BMJ. 2001; 323(7305):171.

15 May MB, Glode AE. Dronabinol for chemotherapy-induced nausea and vomiting unresponsive to antiemetics. Cancer Manag Res. 2016;8:49-55.
16 Roncoroni AJ. [Medical use of cannabis and synthetic cannabinoids]. Medicina. 2003; 63(6):748-52. Spanish.

17 Health products containing cannabis or for use with cannabis: guidance for the Cannabis Act, the Food and Drugs Act, and related regulations. Government of Canada; 2020 May 21. Available from: https://www.canada.ca/ en/health-canada/services/drugs-healthproducts/drug-products/applications-submissions/guidance-documents/guidancecannabis-act-food-and-drugs-act-relatedregulations/document.html Accessed 2020 Sep 24.

18 Barrus DG, Capogrossi KL, Cates SC, Gourdet CK, Peiper NC, Novak SP, et al. Tasty THC: promises and challenges of cannabis edibles. Methods Rep RTI Press. 2016;2016: 10.3768/rtipress.2016.op.0035.161.

19 Russo EB. Cannabinoids in the management of difficult to treat pain. Ther Clin Risk Manag. 2008;4(1):245-59.

20 Karchner LE, Darwin DW, Goodwin SR, Wright S, Huestis AM. Plasma cannabinoid pharmacokinetics following controlled oral $\Delta$ 9-tetrahydrocannabinol and oromucosal cannabis extract administration. Clin Chem. 2011;57(1):66-75.

21 Namaka M, Leong C, Grossberndt A, Klowak M, Turcotte D, Esfahani F, et al. A treatment algorithm for neuropathic pain: an update. Consult Pharm. 2009;24(12):885-902.

22 Pertwee RG, Howlett AC, Abood ME, Alexander SPH, Di Marzo V, Elphick MR, et al. International union of basic and clinical pharmacology. LXXIX. Cannabinoid receptors and their ligands: beyond CB1 and CB2. Pharmacol Rev. 2010;62(4):588-631.

23 Überall MA. A review of scientific evidence for THC:CBD oromucosal spray (Nabiximols) in the management of chronic pain. J Pain Res. 2020;13:399-410.

24 Brown MRD, Farquhar-Smith WP. Cannabinoids and cancer pain: a new hope or a false dawn? Eur J Intern Med. 2018;49:30-6.

25 Ibrahim MM, Porreca F, Lai J, Albrecht PJ, Rice FL, Khodorova A, et al. CB2 cannabinoid receptor activation produces antinociception by stimulating peripheral release of endogenous opioids. Proc Natl Acad Sci U S A. 2005; 102(8):3093-8.

26 Dubin AE, Patapoutian A. Nociceptors: the sensors of the pain pathway. J Clin Invest. 2010;120(11):3760-72.

27 Di Forti M, Marconi A, Carra E, Fraietta S, Trotta A, Bonomo M, et al. Proportion of patients in South London with first-episode psychosis attributable to use of high potency cannabis: a case - control study. Lancet Psychiat. 2015;2(3):233-8.

28 Morgan DR. Therapeutic uses of cannabis. Amsterdam: Harwood Academic; 1997.

29 McInnis OA, Porathller A. Chronic use of cognitive functioning and mental health: an update. Canadian Centre on Substance
Abuse; 2016. Available from: http://www. ccsa.ca/Resource\%20Library/CCSA-Chronic-Cannabis-Use-Effects-Report-2016-en.pdf Accessed 2020 Sep.

30 Deshpande A, Mailis-Gagnon A, Zoheiry N, Lakha SF. Efficacy and adverse effects of medical marijuana for chronic noncancer pain: systematic review of randomized controlled trials. Can Fam Physician. 2015;61(8):e37281.

31 Wilkinson ST, Stefanovics E, Rosenheck RA. Marijuana use is associated with worse outcomes in symptom severity and violent behavior in patients with posttraumatic stress disorder. J Clin Psychiatry. 2015;76(9):117480.

32 Massi P, Solinas M, Cinquina V, Parolaro D. Cannabidiol as potential anticancer drug. $\mathrm{Br} J$ Clin Pharmacol. 2013;75(2):303-12.

33 Authorizing dried cannabis for chronic pain or anxiety: preliminary guidance. 2014 Sep. Available from: https://portal.cfpc.ca/resourcesdocs/uploadedFiles/Resources/_ PDFs/Authorizing\%20Dried\%20Cannabis\%20for\%20Chronic\%20Pain\%20or\%20 Anxiety.pdf Accessed 2020 Sep.

34 Diarrhea: causes, treatment, and symptoms. Medical News Today; 2020 May 31. Available from: https://www.medicalnewstoday.com/ articles/158634 Accessed 2020 Sep.

35 Sun S, Zimmermann AE. Cannabinoid hyperemesis syndrome. Hosp Pharm. 2013; 48(8):650-5.

36 Jones RT. Cardiovascular system effects of marijuana. J Clin Pharmacol. 2002;42(S1): 58S-63S.

37 Aryana A, Williams MA. Marijuana as a trigger of cardiovascular events: speculation or scientific certainty? Int J Cardiol. 2007;118(2): 141-4.

38 Śledziński P, Zeyland J, Słomski R, Nowak A. The current state and future perspectives of cannabinoids in cancer biology. Cancer Med. 2018;7(3):5859-75

39 Shmagel A, Krebs E, Ensrud K, Foley R. Illicit substance use in US adults with chronic low back pain. Spine. 2016;41(17):1372-7.

40 Abuhasira R, Schleider LB, Mechoulam R, Novack V. Epidemiological characteristics, safety and efficacy of medical cannabis in the elderly. Eur J Intern Med. 2018;49:4450.

41 Hartung B, Kauferstein S, Ritz-Timme S, Daldrup T. Sudden unexpected death under acute influence of cannabis. Forensic Sci Int. 2014;237:e11-3.

42 Information for health care professionals: cannabis (marihuana, marijuana) and the cannabinoids. 2018. Available from: https:// www.canada.ca/en/health-canada/services/ drugs-health-products/medical-use-marijuana/information-medical-practitioners/information-health-care-professionals-cannabismarihuana-marijuana-cannabinoids.html Accessed 2020 Sep. 
43 Maida V, Daeninck PJ. A user's guide to cannabinoid therapies in oncology. Curr Oncol. 2016;23(6):398-406.

44 Mücke M, Phillips T, Radbruch L, Petzke F, Häuser W. Cannabis-based medicines for chronic neuropathic pain in adults. Cochrane Database Syst Rev. 2018;3(3):CD012182.

45 Hoggart B, Ratcliffe S, Ehler E, Simpson KH, Hovorka J, Lejčko J, et al. A multicentre, open-label, follow-on study to assess the longterm maintenance of effect, tolerance and safety of THC/CBD oromucosal spray in the management of neuropathic pain. J Neurol. 2015;262(1):27-40.

46 Issa MA, Narang S, Jamison RN, Michna E, Edwards RR, Penetar DM, et al. The subjective psychoactive effects of oral dronabinol studied in a randomized, controlled crossover clinical trial for pain. Clin J Pain. 2014;30(6): $472-8$.

47 Turcotte D, Doupe M, Torabi M, Gomori A, Ethans K, Esfahani F, et al. Nabilone as an adjunctive to gabapentin for multiple sclerosisinduced neuropathic pain: a randomized controlled trial. Pain Med. 2015;16(1):149-59.

48 Busse JW, Wang L, Kamaleldin M, Craigie S, Riva JJ, Montoya L, et al. Opioids for chronic noncancer pain: a systematic review and meta-analysis. JAMA. 2018;320(23):2448-60.

49 Capano A, Weaver R, Burkman E. Evaluation of the effects of CBD hemp extract on opioid use and quality of life indicators in chronic pain patients: a prospective cohort study. Postgrad Med. 2020;132(1):56-61.

50 Ware MA, Wang T, Shapiro S, Collet JP. Cannabis for the management of pain: assessment of safety study (COMPASS). J Pain. 2015; 16(12):1233-42.

51 Eisenberg E, Ogintz M, Almog S. The pharmacokinetics, efficacy, safety, and ease of use of a novel portable metered-dose cannabis inhaler in patients with chronic neuropathic pain: a phase 1a study. J Pain Palliat Care Pharmacother. 2014;28(3):216-25.

52 Wendelmuth C, Wirz S, Torontali M, Gastmeier A, Gastmeier K. Dronabinol in geriatric pain and palliative care patients: a retrospective evaluation of statutory-health-insurancecovered outpatient medical treatment. Schmerz. 2019;33(5):384-91.
53 Cameron C, Watson D, Robinson J. Use of a synthetic cannabinoid in a correctional population for posttraumatic stress disorder-related insomnia and nightmares, chronic pain, harm reduction, and other indications: a retrospective evaluation. J Clin Psychopharmacol. 2014;34(5):559-64.

54 Russo M, Naro A, Leo A, Sessa E, D’Aleo G, Bramanti $\mathrm{P}$, et al. Evaluating sativex ${ }^{\circledR}$ in neuropathic pain management: a clinical and neurophysiological assessment in multiple sclerosis. Pain Med. 2016;17(6):1145-54.

55 Cuñetti L, Manzo L, Peyraube R, Arnaiz J, Curi L, Orihuela S. Chronic pain treatment with cannabidiol in kidney transplant patients in uruguay. Transplant Proc. 2018; 50(2):461-4.

56 Maida V, Corban J. Topical medical cannabis: a new treatment for wound pain-three cases of pyoderma gangrenosum. J Pain Symptom Manage. 2017;54(5):732-6.

57 Yeung B, Patel P, Nelson A, Dayal R. Cannabidiol in the geriatric population: a novel treatment option for chronic back pain. Pain Physician. 2018;21(3):E294.

58 Bruce D, Brady JP, Foster E, Shattell M. Preferences for medical marijuana over prescription medications among persons living with chronic conditions: alternative, complementary, and tapering uses. J Altern Complement Med. 2018;24(2):146-53.

59 Walitt B, Klose P, Fitzcharles MA, Phillips T, Häuser W. Cannabinoids for fibromyalgia. Cochrane Database Syst Rev. 2016;7(7): CD011694.

60 Andreae MH, Carter GM, Shaparin N, Suslov $\mathrm{K}$, Ellis RJ, Ware MA, et al. Inhaled cannabis for chronic neuropathic pain: a meta-analysis of individual patient data. J Pain. 2015;16(12): 1221-32.

61 Hickernell TR, Lakra A, Berg A, Cooper HJ, Geller JA, Shah RP. Should cannabinoids be added to multimodal pain regimens after total hip and knee arthroplasty? J Arthroplasty. 2018;33(12):3637-41.
62 Liu CW, Bhatia A, Buzon-Tan A, Walker S, Ilangomaran D, Kara J, et al. Weeding out the problem: the impact of preoperative cannabinoid use on pain in the perioperative period. Anesth Analg. 2019;129(3):874-81.

63 Fitzcharles M-A, Baerwald C, Ablin J, Häuser W. Efficacy, tolerability and safety of cannabinoids in chronic pain associated with rheumatic diseases (fibromyalgia syndrome, back pain, osteoarthritis, rheumatoid arthritis). Schmerz. 2016;30(1):47-61.

64 van den Elsen GA, Ahmed AI, Lammers M, Kramers C, Verkes RJ, van der Marck MA, et al. Efficacy and safety of medical cannabinoids in older subjects: a systematic review. Ageing Res Rev. 2014;14:56-64.

65 Meng H, Johnston B, Englesakis M, Moulin DE, Bhatia A. Selective cannabinoids for chronic neuropathic pain: a systematic review and meta-analysis. Anesth Analg. 2017; 125(5):1638-52.

66 Brazier Y. Multiple sclerosis: what you need to know. Medical News Today; 2019 May 29. Available from: https://www.medicalnewstoday.com/articles/37556 Accessed Sep 2020.

67 Velayudhan L, McGoohan K, Bhattacharyya S. Safety and tolerability of natural and synthetic cannabinoids in adults aged over 50 years: a systematic review and meta-analysis. PLoS Med. 2021;18(3):e1003524.

68 Carter GT, Javaher SP, Nguyen MH, Garret S, Carlini BH. Re-branding cannabis: the next generation of chronic pain medicine? Pain Manag. 2015;5(1):13-21.

69 Bestard JA, Toth CC. An open-label comparison of nabilone and gabapentin as adjuvant therapy or monotherapy in the management of neuropathic pain in patients with peripheral neuropathy. Pain Pract. 2011;11(4):35368.

70 Lynch ME, Cesar-Rittenberg P, Hohmann AG. A double-blind, placebo-controlled, crossover pilot trial with extension using an oral mucosal cannabinoid extract for treatment of chemotherapy-induced neuropathic pain. J Pain Symptom Manage. 2014;47(1): 166-73. 